\title{
Derive boundary conditions for holistic discretisations of Burgers' equation
}

\author{
A. J. Roberts* \\ (Received 26 June 2001)
}

\begin{abstract}
I previously used Burgers' equation to introduce a new method of numerical discretisation of PDEs. The analysis is based upon centre manifold theory so we are assured that the discretisation accurately models all the processes and their subgrid scale interactions. Here I show how boundaries to the physical domain may be naturally incorporated into the numerical modelling of Burgers' equation. We investigate Neumann and Dirichlet boundary conditions. As well as modelling the nonlinear advection, the method naturally derives symmetric matrices with constant bandwidth to correspond to the self-adjoint diffusion operator. The techniques developed here may be used to accurately model the nonlinear evolution of quite general spatio-temporal dynamical systems on bounded domains.
\end{abstract}

*Dept. Maths \& Computing, University of Southern Queensland, Toowoomba, Queensland 4352, AUstralia. mailto: aroberts@usq.edu. au ${ }^{0}$ See http://anziamj . austms . org. au/V44/CTAC2001/Robe for this article, (C) Austral. Mathematical Soc. 2003. Published 1 April 2003. ISSN 1446-8735 


\section{Contents}

1 Introduction

C665

2 Holistic discretisation of Burgers' equation in the interior

C667

3 Dirichlet boundary conditions applied at a grid point

C673

4 Apply Neumann boundary conditions at a midpoint

C678

5 Conclusion

C681

A Computer algebra derives different boundary discretisations

C682

References

C685

\section{Introduction}

We discuss the discretisation of boundary conditions for partial differential equations. The holistic discretisation [7] we use herein is based upon the support centre manifold theory gives to the nonlinear dynamics on finite grid spacing. We expect such discretisation will have good stability and high accuracy on coarse grids because it systematically accounts for subgrid scale interactions. To date we have considered periodic problems [7, 3, 4, 9] and their initial conditions [8]. Here we show how to incorporate a couple of boundary conditions into the analysis. 
Study Burgers' equation: As an illustrative example we restrict attention to the one-dimensional spatial discretisation of Burgers' equation

$$
\frac{\partial u}{\partial t}+u \frac{\partial u}{\partial x}=\frac{\partial^{2} u}{\partial x^{2}},
$$

which contains the important mechanisms of diffusion, $u_{x x}$, and nonlinear advection, $u u_{x}$. As example boundary conditions we consider the important cases of Dirichlet fixed field, §3, and Neumann fixed flux boundary conditions, $\S 4$. The same techniques may be straightforwardly extended to other partial differential equations, other boundary conditions and higher spatial dimensions.

Discretise on finite size elements: The analysis is based upon dividing the domain into finite sized elements, each separated from their two neighbours by specially crafted artificial internal boundary conditions (IBCs). The form of the IBCs (3-4) generates a discretisation in the interior of the domain (§2) which is not only linearly consistent, as proved in [9], but which appears to be also nonlinearly consistent to high order. This observation is based upon this analysis of Burgers' equation and work in progress on the KuramotoSivashinsky equation; further research is needed to prove it in general.

Focus on boundary conditions: Here we investigate the discretisation near the boundary of the domain. Boundary conditions are incorporated simply by replacing an IBC of an end element by a variant of the actual boundary condition: the Dirichlet boundary condition of fixed field $u$ is implemented as (11); the Neumann boundary condition of fixed flux $u_{x}$ is (19). The computer algebra of $\S$ A readily computes the effect these boundary conditions have 
on the discretisation near the boundary. Novel features of this dynamical systems approach are the following:

- theoretical support is provided on finite grid spacing $h$ as explained in $[7,9$, e.g.];

- the discretisation has a consistent bandwidth across the whole domain including near the boundaries;

- high order discretisations are expressed in terms of the matrices of the basic, second order, centred difference operators, leading to appropriately symmetric discretisations of the diffusion operator, for example;

- time variations of boundary values, say $a(t)$, not only has a direct effect but also involves time derivative factors, such as $\dot{a}$, which are more important on coarser grids due to the time it takes changes in a boundary value to diffuse into the element.

\section{Holistic discretisation of Burgers' equation in the interior}

We consider Burgers' equation (1) on some finite domain in $x$. Place grid points $x_{j}$ equi-spaced across the domain with constant spacing $h$, and correspondingly define $u_{j}=u\left(x_{j}, t\right)$, that is, $u_{j}$ is the evolving field $u$ evaluated at the $j$ th grid point. Divide the domain into $m$ elements with a grid point at the centre of each. Following [9] to apply the theory we artificially create each element using internal boundary conditions (IBCs) in the discrete form

$$
\mu_{x} \delta_{x} v_{j}(x, t)=\gamma \mu \delta u_{j} \quad \text { and } \quad \delta_{x}^{2} v_{j}(x, t)=\gamma \delta^{2} u_{j},
$$


evaluated at $x=x_{j}$ where $v_{j}$ denotes the field in the $j$ th element. Throughout this work we use discrete operators [5, p65, e.g.]:

- shift operator, $E u_{j}=u_{j+1}$ and $E_{x} u=u(x+h, t)$.

- centred difference, $\delta u_{j}=\left(E^{1 / 2}-E^{-1 / 2}\right) u_{j}=u_{j+1 / 2}-u_{j-1 / 2}$ and $\delta_{x} u=\left(E_{x}^{1 / 2}-E_{x}^{-1 / 2}\right) u=u(x+h / 2, t)-u(x-h / 2, t)$;

- and centred mean, $\mu u_{j}=\frac{1}{2}\left(E^{1 / 2}+E^{-1 / 2}\right) u_{j}=\frac{1}{2}\left[u_{j+1 / 2}+\right.$ $\left.u_{j-1 / 2}\right]$ and $\mu_{x} u=\frac{1}{2}\left(E_{x}^{1 / 2}+E_{x}^{-1 / 2}\right) u=\frac{1}{2}[u(x+h / 2, t)+u(x-$ $h / 2, t)]$.

Thus, for example, $\mu \delta u_{j} /(2 h)$ approximates the first derivative at $x_{j}$, whereas $\delta^{2} u_{j} / h^{2}$ approximates the second derivative. As shown in [9], the particular choice of IBCs (2) ensures that the resultant finite difference scheme is consistent to high order in $h$ as the grid size $h \rightarrow 0$. In this section we repeat the construction of the holistic discretisation, following [7], away from the domain boundaries via centre manifold theory but with a new and convenient form of these discrete IBCs.

Internal boundary conditions: In actually developing discrete models the IBCs may take any of many equivalent forms [7, e.g.]. In later sections we investigate the discretisation near a boundary of the domain: the element adjacent a boundary has one real boundary and one artificial internal boundary. Thus it is appropriate to rewrite the two IBCs in (2) in the form of two conditions, one at the left edge of each internal element, and one at the right. Recall that the difference operators $\mu \pm \frac{1}{2} \delta=E^{ \pm 1 / 2}$ [5, p65, e.g.] so that to the first IBC in (2) add/subtract half the second to give the equivalent IBCS

$$
\delta_{x} v_{j}(x, t)=\gamma \delta u_{j-1 / 2} \text { at } x=x_{j-1 / 2},
$$




$$
\text { and } \quad \delta_{x} v_{j}(x, t)=\gamma \delta u_{j+1 / 2} \quad \text { at } x=x_{j+1 / 2} .
$$

The IBC (4) is to hold at the right-hand side of each element and the IBC (3) is to hold at the left. The introduced parameter $\gamma$, when non-zero, couples each element to its neighbour. When $\gamma=0$ these IBCs effectively insulate each element from its neighbours and forms the basis of the centre manifold analysis; whereas when $\gamma=1$ they assert that the field in the $j$ th element when extended into the surrounding elements has the same differences centred across each internal boundary as that given by the grid point values. Thus evaluating the model at $\gamma=1$ forms the relevant discretisation. These IBCs apply to all elements except for the leftmost and rightmost elements, the ones adjacent to the boundary: in the leftmost element the left-hand IBC, (3) with $j=1$, is replaced by the actual boundary condition; in the rightmost element the right-hand IBC, (4) with $j=m$, is replaced by the actual boundary condition. In this paper I analyse the left boundary of the domain - discretisations near the right boundary are similar by symmetry.

Centre manifold theory supports discretisation: the theory addresses linear and nonlinear perturbations of linear dynamical systems. Here the nonlinear advection $u u_{x}$ and the coupling linear IBCs (3-4) perturb the linear dynamics of diffusion, $u_{t}=u_{x x}$, within insulated finite elements. Abstractly the theory [1, 2, e.g.] considers dynamics in the form

$$
\boldsymbol{u}_{t}=\mathcal{L} \boldsymbol{u}+\boldsymbol{f}(\boldsymbol{u}, \gamma)
$$

where $\boldsymbol{u}(t)$ is the evolving system state, $\mathcal{L}$ is a linear operator, and $\boldsymbol{f}$ are the perturbation terms. If about any fixed point, usually taken to be $\boldsymbol{u}=\mathbf{0}$, the spectrum of the linear operator $\mathcal{L}$ is composed of $m$ eigenvalues with zero real part, and the rest of the spectrum has negative real part and is bounded away from zero, say less than $-\beta$, 
then centre manifold theory generates useful models of the dynamics. Here, the spectrum of linear diffusion within each element is one zero eigenvalue, corresponding to conservation of $u$ in each insulated element, and the other eigenvalues less than $-\beta=-\pi^{2} /\left(4 h^{2}\right)$. Hence we know that, linearly, all initial conditions decay exponentially quickly to a state where all $m$ conserved neutral modes are constant, and all other modes zero. Centre manifold theory asserts that in the presence of perturbations, this is still true but that the decaying modes are forced by the neutral modes through the perturbations and that the neutral modes evolve slowly through the coupling of the perturbations. We invoke the following three centre manifold theorems (described rather roughly for simplicity). Given the system (5) with linear operator $\mathcal{L}$ as described above and smooth enough perturbation $\boldsymbol{f}$, then:

1. in some neighbourhood of the origin, an $m$-dimensional centre manifold exists parametrised by amplitudes of the $m$ neutral modes and the amplitudes evolve in time. Here, in the $j$ th element the field

$$
u(x, t)=v_{j}\left(\boldsymbol{u}_{\boldsymbol{j}}, x, \gamma\right) \text { such that } \dot{u}_{j}=g_{j}\left(\boldsymbol{u}_{\boldsymbol{j}}, \gamma\right) ;
$$

that is, a dynamic model exists in terms of the grid values $u_{j}$, a model which resolves the subgrid scale field, $u(x, t)=$ $v_{j}\left(\boldsymbol{u}_{j}, x, \gamma\right)$.

2. further, the model is relevant because all bounded solutions in a neighbourhood of the centre manifold are exponentially quickly attracted to a solution of the model, like $\mathcal{O}\left(e^{-\beta^{\prime} t}\right)$ where $\beta^{\prime} \approx \beta$. Thus here if we construct the centre manifold accurately enough we know that our model would genuinely capture all the bounded long term dynamics of the original system. This applies at finite grid spacing $h$ and is a novel guarantee of the fidelity of a discretisation. 
3. lastly, the expressions (6) describing the centre manifold and the evolution thereon may be found simply by substituting (6) into the original system (5), then solving to some asymptotic order. The error in the expressions is then of the same order as the residuals of the equation.

These are the key centre manifold theorems of existence, relevance and construction. Here they guarantee a discretisation exists which completely models the long term dynamics, and which we can find by approximately solving Burgers' equation through (6). The only caveat is that in this application we have to evaluate the perturbing influences at $\gamma=1$ whereas the theory only asserts that there is a neighbourhood of $\gamma=0$ in which the results hold. However, for many asymptotic expansions, one is a small enough number.

The interior is straightforward: In the interior of the domain the physical boundaries have no influence upon the discretisation. Each element in the interior has IBCs (3) and (4). Executing the computer algebra program in Appendix A, adapted from [7], the subgrid structure of the solution field $u(x, t)$ is, in terms of $\xi=$ $\left(x-x_{j}\right) / h$,

$$
\begin{aligned}
v_{j}= & u_{j}+\gamma\left[\xi \mu \delta u_{j}+\frac{1}{2} \xi^{2} \delta^{2} u_{j}\right] \\
& +\gamma^{2}\left[\frac{1}{6}\left(\xi^{3}-\xi\right) \mu \delta^{3} u_{j}+\frac{1}{24}\left(\xi^{4}-\xi^{2}\right) \delta^{4} u_{j}\right] \\
& +\gamma h \frac{1}{6}\left(\xi^{3}-\xi\right) u_{j} \delta^{2} u_{j}+\mathcal{O}\left(\|\boldsymbol{u}\|^{4}+\gamma^{4}\right) .
\end{aligned}
$$

This expression gives the field $u$ everywhere in the interior as a function of the grid values $u_{j}$-it describes the low-dimensional centre manifold in the original state space. The inter-element coupling and the nonlinearities cause these grid values to evolve. By requiring Burgers' equation (1) be satisfied to some order of perturbation, 
the evolution on this centre manifold forms the holistic discretisation in the interior to the same order of error:

$$
\begin{aligned}
\dot{u}_{j}= & \frac{1}{h^{2}}\left[\gamma \delta^{2} u_{j}-\frac{\gamma^{2}}{12} \delta^{4} u_{j}+\frac{\gamma^{3}}{90} \delta^{6} u_{j}\right] \\
& -\frac{1}{h} u_{j}\left[\gamma \mu \delta u_{j}-\frac{\gamma^{2}}{6} \mu \delta^{3} u_{j}\right]+\frac{\gamma^{2}}{24 h}\left(\delta^{2} u_{j} \mu \delta^{3} u_{j}+\delta^{4} u_{j} \mu \delta u_{j}\right) \\
& +\frac{\gamma}{12} u_{j}^{2} \delta^{2} u_{j}+\mathcal{O}\left(\|\boldsymbol{u}\|^{5}+\gamma^{5}\right) .
\end{aligned}
$$

As discussed previously [7] when the coupling parameter $\gamma=1$ : the first line gives successive approximations to the diffusion term $u_{x x}$; the second line gives approximations to the nonlinear advection; and lastly, the cubic nonlinear term, $u_{j}^{2} \delta^{2} u_{j}$ in the last line above, accounts for subgrid scale interactions between the advection and diffusion and acts to stabilise the numerical model. Higher-order terms are easily found by the computer algebra of Appendix A but for clarity are not presented here.

These discretisations are also consistent: In the limit as the grid spacing $h \rightarrow 0$ higher-order discretisations have the equivalent PDE

$$
\begin{aligned}
& \frac{\partial u}{\partial t}=\gamma\left[-u \frac{\partial u}{\partial x}+\frac{\partial^{2} u}{\partial x^{2}}\right]+\frac{h^{2}}{12} \gamma(1-\gamma)\left[u_{x x x x}-2 u u_{x x x}+u^{2} u_{x x}\right] \\
&+\frac{h^{4}}{720} \gamma(1-\gamma)\left[2 \gamma \left(-5 u_{x}^{2} u_{x x}-9 u u_{x x}^{2}-25 u u_{x} u_{x x x}\right.\right. \\
&\left.+15 u_{x x} u_{x x x}+15 u_{x} u_{x x x x}-2 u^{2} u_{x x x x}\right) \\
&+ \\
&\left.+(1-4 \gamma)\left(2 \partial_{x}^{6} u-6 u \partial_{x}^{5} u+5 u^{2} \partial_{x}^{4} u\right)\right] \\
&+ \mathcal{O}\left(\|u\|^{4}, \gamma^{4}, h^{6}\right)
\end{aligned}
$$

See that upon substituting $\gamma=1$ to recover a discretisation for the Burgers' equation (1), we would find an equivalent PDE to an error 
$\mathcal{O}\left(h^{6},\|u\|^{4}\right)$. Analogous lower order discretisations are obtained, as promised by the analysis in [9], when we truncate the discretisation (8) to lower orders in the coupling parameter $\gamma$. But observe the new feature that the discrete form (3-4) of the IBCs lead to discretisations which are not only linearly consistent, as assured in [9], but are also nonlinearly consistent. Further research is needed to establish nonlinear consistency in general.

\section{$3 \quad$ Dirichlet boundary conditions applied at a grid point}

Consider the case of Dirichlet conditions on the boundary of the domain of prescribed $u$ at a grid point; without loss of generality say

$$
u=a(t) \quad \text { at } x=x_{0} .
$$

This boundary condition is included in the analysis simply by replacing the left-hand IBC in the leftmost element, (3) with $j=1$, by (as if $u_{0}=a$ in (3))

$$
\delta_{x} v_{1}(x, t)=\gamma\left(u_{1}-a\right) \quad \text { at } x=x_{1 / 2} .
$$

(Implicitly the first element then extends from $x_{0}=x_{1}-h$ to $x_{1}+$ $h / 2$.) When the coupling parameter $\gamma=0$ this IBC effectively insulates the first element from the conditions at the domain boundary. However, when $\gamma=1$, since $v_{j}\left(x_{j}, t\right)=u_{j}$, this reduces to (10) by requiring $v_{1}\left(x_{0}, t\right)=a$. Hence, the centre manifold derivation is based on $\gamma=0$ as explained in [7] and evaluated at $\gamma=1$ to obtain a discretisation of Burgers' equation.

Evolution near the boundary: We solve for the subgrid fields and evolution in the elements near the boundary, $j=1,2, \ldots$ The 
computer algebra program in Appendix A implements this boundary condition when dirichlet: $=1$. The influence of this specified boundary value affects a number of elements near the boundary equal to the order of $\gamma$ retained in the analysis, nothing else: in the interior the discretisation is (8); whereas for elements the near the boundary and for errors $\mathcal{O}\left(\|\boldsymbol{u}\|^{5}+\gamma^{5}\right)$ we find the evolution to be of the form

$$
\begin{aligned}
{\left[\begin{array}{c}
\dot{u}_{1} \\
\dot{u}_{2} \\
\dot{u}_{3}
\end{array}\right]=} & \frac{1}{h^{2}}\left(\mathcal{D} \boldsymbol{u}+\boldsymbol{f}_{d}\right)-\frac{1}{h}\left(U \mathcal{C} \boldsymbol{u}+\boldsymbol{g}_{c}(\boldsymbol{u})+\boldsymbol{f}_{c}\right) \\
& +\left(U^{2} \mathcal{B} \boldsymbol{u}+\boldsymbol{f}_{b}\right)+\mathcal{O}\left(\|\boldsymbol{u}\|^{5}+\gamma^{5}\right)
\end{aligned}
$$

where $U=\operatorname{diag}\left(u_{1}, u_{2}, u_{3}\right)$ is the diagonal matrix of grid velocities and the three parts of the right-hand side represent respectively the discretisation of the diffusion, the nonlinear advection, and the leading order interaction between advection and diffusion. These parts include the forcing due to the time dependent boundary value $a$. Here the various terms are found to be:

$$
\begin{aligned}
\mathcal{D}=\gamma & {\left[\begin{array}{rrrrr}
-2 & 1 & & \\
1 & -2 & 1 & & \ldots \\
1 & -2 & 1 &
\end{array}\right] } \\
& -\frac{\gamma^{2}}{12}\left[\begin{array}{rrrrrr}
5 & -4 & 1 & & \\
-4 & 6 & -4 & 1 & \ldots \\
1 & -4 & 6 & -4 & 1 &
\end{array}\right] \\
& +\frac{\gamma^{3}}{90}\left[\begin{array}{rrrrrr}
-14 & 14 & -6 & 1 & & \\
14 & -20 & 15 & -6 & 1 & \ldots \\
-6 & 15 & -20 & 15 & -6 & 1
\end{array}\right]+\mathcal{O}\left(\gamma^{4}(13)\right. \\
\mathcal{C}= & \frac{\gamma}{2}\left[\begin{array}{rrrr}
0 & 1 & & \\
0 & 1 & & \ldots \\
-1 & 0 & 1 &
\end{array}\right]
\end{aligned}
$$




$$
\begin{aligned}
& -\frac{\gamma^{2}}{12}\left[\begin{array}{rrrrrr}
0 & -2 & 1 & & & \\
2 & 0 & -2 & 1 & & \cdots \\
-1 & 2 & 0 & -2 & 1 &
\end{array}\right] \\
& +\mathcal{O}\left(\gamma^{3}\right) \\
& \boldsymbol{g}_{c}=\frac{\gamma^{2}}{24}\left[\begin{array}{c}
\frac{1}{2} \boldsymbol{u}^{\top} G_{1} \boldsymbol{u} \\
\frac{1}{2} \boldsymbol{u}^{\top} G_{2} \boldsymbol{u} \\
\frac{1}{2} \boldsymbol{u}^{\top} G_{3} \boldsymbol{u}
\end{array}\right] \quad \text { where } \quad G_{1}=\left[\begin{array}{ccc}
2 & 5 & -1 \\
5 & -6 & 1 \\
-1 & 1 & 0
\end{array}\right] \text {, } \\
& G_{2}=\left[\begin{array}{cccc}
6 & -5 & 0 & 0 \\
-5 & 0 & 5 & -1 \\
0 & 5 & -6 & 1 \\
0 & -1 & 1 & 0
\end{array}\right] \text {, } \\
& G_{3}=\left[\begin{array}{ccccc}
0 & -1 & 1 & 0 & 0 \\
-1 & 6 & -5 & 0 & 0 \\
1 & -5 & 0 & 5 & -1 \\
0 & 0 & 5 & -6 & 1 \\
0 & 0 & -1 & 1 & 0
\end{array}\right]
\end{aligned}
$$

and $\mathcal{B}=\frac{1}{12} \mathcal{D}+\mathcal{O}(\gamma)$. Denote by $\mathbb{D}$ the matrix appearing above in $\mathcal{D}$ linear in $\gamma$ and then row extended across the interior of the domain: $\mathbb{D}$ is the matrix of the second-order centred approximation to the second derivative, $\delta^{2}$. Observe that the order $\gamma^{2}$ and $\gamma^{3}$ matrices in $\mathcal{D}$ simply correspond to $\mathbb{D}^{2}$ and $\mathbb{D}^{3}$. Thus the discretisation of the diffusion term, across the entire domain including the near boundary elements, is simply $\gamma \delta^{2}-\frac{\gamma^{2}}{12} \delta^{4}+\frac{\gamma^{3}}{90} \delta^{6}$ seen in the first line of the interior discretisation, (8), but with the matrix $\mathbb{D}$ replacing the centred difference $\delta^{2}$. Thus this approach generates an appropriately symmetric discretisation of the self-adjoint diffusion term $u_{x x}$. The nonlinear stabilisation term, $U^{2} \mathcal{B} \boldsymbol{u}$, also corresponds to replacing $\delta^{2}$ in $(8)$ by $\mathbb{D}$. Similarly, denote by $\mathbb{C}$ the matrix appearing above in $\mathcal{C}$ linear in $\gamma$ (including the factor $\frac{1}{2}$ ) and then row extended across the interior of the domain: $\mathbb{C}$ is the matrix of the second-order centred approximation to the first derivative, $\mu \delta$. Observe that the order $\gamma^{2}$ matrix in $\mathcal{C}$ is $(\mathbb{D} \mathbb{C}+\mathbb{C D}) / 2$ corresponding to the average 
of different permutations of the centred difference operators $\mu \delta^{3}$. Thus again the discretisation of the advection terms across the entire domain is the interior discretisation, $\gamma \mu \delta-\frac{\gamma^{2}}{6}\left(\mu \delta \delta^{2}+\delta^{2} \mu \delta\right) / 2$, with the truncated $\mathbb{C}$ and $\mathbb{D}$ replacing the difference operators $\mu \delta$ and $\delta^{2}$ respectively. Additional terms represented by $\boldsymbol{g}_{c}$ for the discretisation of the advection correspond to the nonlinear higher order term $\frac{\gamma^{2}}{24 h}\left(\delta^{2} u_{j} \mu \delta^{3} u_{j}+\delta^{4} u_{j} \mu \delta u_{j}\right)$ in the model (8). These are pleasing patterns.

The discretisation has constant bandwidth: See that by truncating to a fixed power of the inter-element coupling parameter $\gamma$ we obtain a discretisation that has constant bandwidth across the whole domain. This constant bandwidth will always be derived in this holistic approach, see for another example the Neumann boundary conditions in the next section, because the truncation at a fixed power of the inter-element coupling $\gamma$ controls how many neighbouring elements interact with any given element. Although there is a lower order (in $h$ ) of consistency near the domain boundaries, the support by centre manifold theory is the same across the whole domain. As discussed in [7], the reason for this support is that the theory applies to the solutions of Burgers' PDE (1) in the entire domain, not just in some locale.

Boundary forcing lags on finite grids: Now consider the forcing from the boundary. Using $\boldsymbol{a}=\left(a, h^{2} \dot{a}\right)$, it is

$$
\boldsymbol{f}_{d}=\left[\begin{array}{rr}
\gamma+\frac{\gamma^{2}}{6}+\frac{\gamma^{3}}{18} & -\frac{\gamma}{12}-\frac{\gamma^{2}}{45}-\frac{\gamma^{3}}{112} \\
-\frac{\gamma^{2}}{12}-\frac{2 \gamma^{3}}{45} & +\frac{\gamma^{2}}{90}+\frac{\gamma^{3}}{140} \\
\frac{\gamma^{3}}{90} & -\frac{\gamma^{3}}{560}
\end{array}\right] \boldsymbol{a}+\mathcal{O}\left(\gamma^{4}, \ddot{a}\right),
$$




$$
\begin{aligned}
\boldsymbol{f}_{c}= & \gamma\left[\begin{array}{cc}
\frac{1}{2} u_{1} & -\frac{1}{24} u_{1} \\
0 & 0 \\
0 & 0
\end{array}\right] \boldsymbol{a} \\
& +\frac{\gamma^{2}}{24}\left[\begin{array}{cc}
4 u_{1}+3 a & \frac{1}{15}\left(7 u_{1}-2 u_{2}-9 a\right)+\frac{5}{168} h^{2} \dot{a} \\
-\left(u_{1}+u_{2}\right) & \frac{2}{15}\left(u_{1}+u_{2}\right) \\
0 & 0
\end{array}\right] \boldsymbol{a} \\
& +\mathcal{O}\left(\gamma^{3}, \ddot{a}\right) \\
\boldsymbol{f}_{b}= & \frac{\gamma}{12}\left[\begin{array}{cc}
u_{1}^{2} & -\frac{1}{15} u_{1}^{2} \\
0 & 0 \\
0 & 0
\end{array}\right] \boldsymbol{a}+\mathcal{O}\left(\gamma^{2}, \ddot{a}\right) .
\end{aligned}
$$

See that this holistic approach not only involves the value $a$ of the field at the boundary in the diffusion term, it also involves $a$ in the nonlinear advection (in $\boldsymbol{f}_{c}$ ) and in the nonlinear stabilisation (in $\boldsymbol{f}_{b}$ ). But further, it also involves time derivatives of the boundary value $a$. The reason is clearly that changes in $a$ take time to advect and diffuse into the interior of the first few elements and so the effect of changes in the boundary value $a$ upon the evolution of the grid values will lag, as seen by the opposite sign of the coefficients in the two columns of (16). This lag increases with the element size $h$ and hence accounts for the $h^{2}$ factor multiplying every $\dot{a}$. Similarly, though not recorded above, higher order analysis shows each second derivative appears only in the combination $h^{4} \ddot{a}$. Such effects are important when we try to use, in large scale problems, the expected accuracy and stability of these holistic discretisations on coarse grids. 


\section{Apply Neumann boundary conditions at a midpoint}

Consider PDEs with Neumann boundary conditions of prescribed gradient of the field $u$. Two different sorts of numerical approximations are commonly developed for such a boundary condition: a grid point is placed at the boundary (as for the Dirichlet problem of $\S 3$ ); or the boundary is arranged to be midway between two grid points. For the first case I found that the discretisation of the diffusion is expressed in terms of non-symmetric matrices. For the second case, the matrices are symmetric which again pleasingly corresponds to the self-adjoint nature of the diffusion operator. Thus I report here on the second case where the boundary is at a midpoint of the grid.

Evolution near the boundary: Without loss of generality, let the Neumann boundary condition of prescribed gradient at a grid midpoint be ${ }^{1}$

$$
h \frac{\partial u}{\partial x}=\gamma a(t) \quad \text { at } x=x_{1 / 2},
$$

where, as for the IBCs, we actually are interested in the case $\gamma=1$. To supplement this left-hand boundary condition on the leftmost element we use the IBC (4) as before. We execute the computer algebra program of Appendix A with options dirichlet:=0 and midpoint $:=1 .^{2}$ Again the interior discretisation is (8), whereas in elements the near the boundary we find the grid values evolve ac-

\footnotetext{
${ }^{1}$ Neumann conditions at a grid point, say $x_{1}$, may be incorporated into the analysis by similarly requiring $h \partial u / \partial x=\gamma a$ at $x=x_{1}$ in place of the left-hand IBC (3).

${ }^{2}$ The computer algebra algorithm takes a few more iterations to complete because the algorithm is tuned to the discrete form of the IBC (3) whose lefthand side is only approximately that of (19).
} 
cording to the form (12) where now the matrices are

$$
\begin{aligned}
& \mathcal{D}=\gamma\left[\begin{array}{rrrrr}
-1 & 1 & & & \\
1 & -2 & 1 & & \cdots \\
& 1 & -2 & 1 &
\end{array}\right] \\
& -\frac{\gamma^{2}}{12}\left[\begin{array}{rrrrrr}
2 & -3 & 1 & & & \\
-3 & 6 & -4 & 1 & & \cdots \\
1 & -4 & 6 & -4 & 1 &
\end{array}\right] \\
& +\frac{\gamma^{3}}{90}\left[\begin{array}{rrrrrrr}
-5 & 9 & -5 & 1 & & & \\
9 & -19 & 15 & -6 & 1 & & \cdots \\
-5 & 15 & -20 & 15 & -6 & 1 &
\end{array}\right]+\mathcal{O}\left(\gamma^{4}\right)(20) \\
& \mathcal{C}=\frac{\gamma}{2}\left[\begin{array}{rrrrr}
-1 & 1 & & & \\
-1 & 0 & 1 & & \cdots \\
& -1 & 0 & 1 &
\end{array}\right] \\
& -\frac{\gamma^{2}}{12}\left[\begin{array}{rrrrrr}
1 & -2 & 1 & & & \\
1 & 0 & -2 & 1 & & \ldots \\
-1 & 2 & 0 & -2 & 1
\end{array}\right]+\mathcal{O}\left(\gamma^{3}\right), \\
& \boldsymbol{g}_{c}=\frac{\gamma}{24}\left[\begin{array}{c}
-u_{1}^{2}+u_{2}^{2} \\
0 \\
0
\end{array}\right]+\frac{\gamma^{2}}{24}\left[\begin{array}{c}
\frac{1}{2} \boldsymbol{u}^{\top} G_{1} \boldsymbol{u} \\
\frac{1}{2} \boldsymbol{u}^{\top} G_{2} \boldsymbol{u} \\
\frac{1}{2} \boldsymbol{u}^{\top} G_{3} \boldsymbol{u}
\end{array}\right] \quad \text { where } \\
& G_{1}=\left[\begin{array}{ccc}
-\frac{49}{20} & \frac{19}{15} & -\frac{11}{15} \\
\frac{19}{15} & -\frac{341}{60} & 1 \\
-\frac{11}{15} & 1 & 0
\end{array}\right] \\
& G_{2}=\left[\begin{array}{cccc}
\frac{23}{6} & -\frac{47}{12} & 0 & 0 \\
-\frac{47}{12} & 0 & 5 & -1 \\
0 & 5 & -6 & 1 \\
0 & -1 & 1 & 0
\end{array}\right] \\
& \mathcal{B}=\frac{\gamma}{12} \tilde{\mathbb{D}}+\frac{\gamma}{12}\left[\begin{array}{rrrrr}
-\frac{1}{48} & \frac{1}{48} & & & \\
0 & 0 & 0 & & \ldots \\
& 0 & 0 & 0 &
\end{array}\right]+\mathcal{O}\left(\gamma^{2}\right),
\end{aligned}
$$


and $G_{3}$ is as in (15). Denote by $\tilde{\mathbb{D}}$ the symmetric matrix appearing above in $\mathcal{D}$ linear in $\gamma$ and then row extended across the interior of the domain: $\tilde{\mathbb{D}}$ is the matrix of the second-order centred approximation to the second derivative, $\delta^{2}$, but incorporating a zero derivative condition to the left of the grid. Observe in the order $\gamma^{2}$ and $\gamma^{3}$ matrices of $\mathcal{D}$ that the discretisation near the boundary of the diffusion term, (20), is simply the interior discretisation seen in the first line of (8) with the truncated $\tilde{\mathbb{D}}$ replacing the centred difference $\delta^{2}$. Although here there is no clear pattern in $\mathcal{C}$ nor $\mathcal{B}$, as written above see that: $\mathcal{B}$ is numerically close to $\tilde{\mathbb{D}} / 12$; and, upon denoting $\tilde{\mathbb{C}}$ as the matrix linear in $\gamma$ in $\mathcal{C}$, the $\gamma^{2}$ matrix is $(\tilde{\mathbb{D}} \tilde{\mathbb{C}}+\tilde{\mathbb{C}} \tilde{\mathbb{D}}) / 2$ just as for the Dirichlet boundary conditions. However, in this case the identification is a little forced because numerically small discrepancies have been gathered into $\boldsymbol{g}_{c}$ along with indistinguishable similar terms corresponding to $\frac{\gamma^{2}}{24 h}\left(\delta^{2} u_{j} \mu \delta^{3} u_{j}+\delta^{4} u_{j} \mu \delta u_{j}\right)$ appearing in the interior discretisation (8). Nonetheless the closeness of the match and the appropriate symmetry is reassuring.

Forcing again exhibits memory: The forcing from the boundary takes the form

$$
\begin{aligned}
& \boldsymbol{f}_{d}=\left[\begin{array}{rr}
-\gamma-\frac{\gamma^{2}}{12}-\frac{\gamma^{3}}{45} & +\frac{\gamma}{24}+\frac{11 \gamma^{2}}{1440}+\frac{\gamma^{3}}{378} \\
+\frac{\gamma^{2}}{12}+\frac{\gamma^{3}}{30} & -\frac{11 \gamma^{2}}{1440}-\frac{\gamma^{3}}{252} \\
-\frac{\gamma^{3}}{90} & +\frac{\gamma^{3}}{756}
\end{array}\right] \boldsymbol{a}+\mathcal{O}\left(\gamma^{4}, \ddot{a}\right),(24) \\
& \boldsymbol{f}_{c}=\gamma\left[\begin{array}{cc}
-\frac{11}{24} u_{1} & +\frac{31}{960} u_{1} \\
0 & 0 \\
0 & 0
\end{array}\right] \boldsymbol{a} \\
& +\frac{\gamma^{2}}{24}\left[\begin{array}{cc}
-\frac{1}{6} u_{1}-\frac{11}{60} u_{2}-\frac{199}{120} a & \frac{924 u_{1}-577 u_{2}-3223 a}{10080}-\frac{757}{48384} h^{2} \dot{a} \\
-\left(\frac{11}{12} u_{1}+u_{2}\right) & \frac{53}{480} u_{1}+\frac{11}{120} u_{2} \\
0 & 0
\end{array}\right] \boldsymbol{a}
\end{aligned}
$$




$$
\begin{aligned}
& +\mathcal{O}\left(\gamma^{3}, \ddot{a}\right) \\
& \boldsymbol{f}_{b}=\frac{\gamma}{12}\left[\begin{array}{cc}
-\frac{49}{48} u_{1}^{2} & \frac{637}{5760} u_{1}^{2} \\
0 & 0 \\
0 & 0
\end{array}\right] \boldsymbol{a}+\mathcal{O}\left(\gamma^{2}, \ddot{a}\right)
\end{aligned}
$$

As discussed in the previous section, the boundary value for the flux $a$ appears in a wide range of terms in the discretisation near the boundary. The reason again is that the flux feeds into the subgrid scale fields of the boundary elements and so affects the interaction between the various physical processes. The scope for such interaction increases with increasing element size $h$ and so accounts for the appearance of the $h^{2}$ factor in front of the time derivative $\dot{a}$. We need to know such effects on coarse grids.

\section{Conclusion}

We have considered the discretisation of Burgers' equation (1) on a finite domain as a worked example of incorporating physical boundary conditions into the derivation of holistic discretisations. The two most common physical boundary conditions were considered in $\S 3$ and $\S 4$. Crucially we found: it is easy to maintain the symmetry appropriate to self-adjoint differential operators; discretisations are developed with constant bandwidth across the whole domain; and lastly our resolution of subgrid scale processes shows how time derivatives of the boundary forcing should be included in the discretisation.

Additionally, using the discrete form of the inter-element boundary conditions (3-4) we observe for the first time a high order consistency of the nonlinear dynamics of Burgers' equation. 
Acknowledgements: this research was supported in part by a grant from the Australian Research Council.

\section{A Computer algebra derives different boundary discretisations}

Straightforward computer algebra programs are written to find the centre manifold and the evolution thereon [6, e.g.]. To ensure correctness and to provide a basis for further work I include the computer algebra code. This replaces extensive recording of elementary algebraic steps in the derivation of the results.

I implement the construction algorithm in $\operatorname{REDUCE}^{3}$ The overall plan of the algorithm is to iteratively satisfy Burgers' equation (1) and the relevant internal (3-4) and actual boundary conditions. A general interior element is analysed in lines $47-53$ while the o: $=3$ elements near the boundary are analysed in the loop of lines 5471. Although there are many details in the program, the correctness of the results are only determined by driving to zero (lines 53, 70 and 73) the residuals of Burgers' equation in each element and the internal and domain boundary conditions: lines 47-9 evaluate the residuals for an arbitrary interior element; lines $55-8$ for near domain boundary elements; and lines 60 or 62 the domain boundary condition. The other details, such as the updates computed in lines 50-2 and 67-9, only affect the rate of convergence to the ultimate answer.

1 Comment Find the discretisation of Burgers' equation, or

${ }^{3}$ At the time of writing, information about reduce was available from Anthony C. Hearn, RAND, Santa Monica, CA 90407-2138, USA. mailto: reduce@rand.org 
2 variants, with three different boundary conditions:

$3 *$ Dirichlet, $\mathrm{u}=\mathrm{a}(\mathrm{t})$ at $\mathrm{x}=\mathrm{x}_{-} 0$

$4 *$ Neumann, midpoint, $u_{-} \mathrm{x}=\mathrm{a}(\mathrm{t}) / \mathrm{h}$ at $\mathrm{x}=\mathrm{x}_{-}\{1 / 2\}$

$5 *$ Neumann, gridpoint, $u_{-} \mathrm{x}=\mathrm{a}(\mathrm{t}) / \mathrm{h}$ at $\mathrm{x}=\mathrm{x}_{-} 1$

6 (c) Tony Roberts, May 2001;

$7 \%$ set options to 1 (true) or 0 (false)

8 dirichlet:=1; midpoint: $=0$;

$90:=3 ; \%$ is number of near boundary elements

$11 \%$ improve printing

12 on div; off allfac; on revpri; factor gam,h;

13

$14 \%$ make function of $x i=\left(x-x_{-} j\right) / h$

15 depend $x i, x$;

16 let $\mathrm{df}(\mathrm{xi}, \mathrm{x})=>1 / \mathrm{h}$;

17

$18 \%$ solvability condition

19 operator solg; linear solg;

20 let $\left\{\operatorname{solg}\left(x i^{\sim \sim} p, x i\right)=>\left(1+(-1)^{\wedge} p\right) /(p+2) /(p+1)\right.$

21

22

$23 \%$ solves $\mathrm{v}^{\prime \prime}=$ RHS s.t. $\mathrm{v}(0)=0$ and $\mathrm{v}(+1)=\mathrm{v}(-1)$

24 operator solv; linear solv;

25 let $\left\{\operatorname{solv}\left(x i^{\sim \sim} p, x i\right) \Rightarrow\right.$

26

27

$\left(\mathrm{xi}^{\wedge}(\mathrm{p}+2)-\left(1-(-1)^{\wedge} \mathrm{p}\right) * \mathrm{xi} / 2\right) /(\mathrm{p}+1) /(\mathrm{p}+2)$

28

29

$30 \%$ parametrise with evolving $u(j)$ and boundary forcing a

31 operator $u$; depend $u, t$;

32 let $\{\mathrm{df}(u(\sim k), t)=>\operatorname{sub}(j=k, g j)$

33

34 when (not $\operatorname{fixp}(k))$ or $(f i x p(k)$ and $k>0)$ , $\operatorname{df}(u(\sim k), t)=>g(k)$ when $\operatorname{fixp}(k)$ and $k<=0$ and $k>0\}$; 
$37 \%$ linear solution in jth element

38 array $\mathrm{g}(\mathrm{o}), \mathrm{v}(\mathrm{o})$;

$39 \mathrm{vj}:=\mathrm{u}(\mathrm{j})$;

$40 \mathrm{gj}:=0$;

41 for $j:=1: 0$ do $v(j):=u(j)$;

42

43

$\%$ iterative refinement to specified error

$44 \%$ here work to error $|u|^{\wedge} 4+\operatorname{gam}^{\wedge} 4$ by multiplying advection by ga

45 let $\left\{\operatorname{gam}^{\wedge} 4=>0, \operatorname{df}(a, t, 2)=>0\right\}$;

46 repeat begin $\%$ first do interior elements

47

48

49

50

51

52

53

54

55

56

57

58

59

60

61

62

63

64

65

66

67

68

69

70

71

deq: $=-d f(v j, t)-g a m * v j * d f(v j, x)+d f(v j, x, 2)$; $r b c:=-(\operatorname{sub}(x i=+1, v j)-\operatorname{sub}(x i=0, v j))+\operatorname{gam} *(u(j+1)-u(j))$; $1 b c:=-(\operatorname{sub}(x i=0, v j)-\operatorname{sub}(x i=-1, v j))+\operatorname{gam} *(u(j)-u(j-1))$; $\operatorname{gd}:=(r b c-1 b c) / h ` 2+\operatorname{solg}(\operatorname{deq}, x i)$;

gj: $:=g j+g d$;

$v j:=v j+h \wedge 2 * \operatorname{solv}(-d e q+g d, x i)+x i *(r b c+l b c) / 2$; ok: $=$ if $(\mathrm{deq}=0)$ and $(\mathrm{rbc}=0)$ and $(\mathrm{lbc}=0)$ then 1 else 0 ; for $j:=1: 0$ do begin $\%$ near boundary elements deq: $=-\operatorname{df}(v(j), t)-\operatorname{gam} * v(j) * d f(v(j), x)+d f(v(j), x, 2)$; $r b c:=-(\operatorname{sub}(x i=+1, v(j))-\operatorname{sub}(x i=0, v(j)))+\operatorname{gam} *(u(j+1)-u(j))$; if $j>1$ then $\%$ internal left $B C$

$l b c:=-\operatorname{sub}(x i=0, v(j))+\operatorname{sub}(x i=-1, v(j))+\operatorname{gam} *(u(j)-u(j-1)$ else if dirichlet then \% dirichlet at $x_{-} 0$

$l b c:=-\operatorname{sub}(x i=0, v(1))+\operatorname{sub}(x i=-1, v(1))+\operatorname{gam} *(u(1)-a)$

else if midpoint then $\%$ neumann at $x_{-}\{1 / 2\}$

lbc: $=-\operatorname{sub}(\mathrm{xi}=-1 / 2, \mathrm{~h} * \mathrm{df}(\mathrm{v}(1), \mathrm{x}))+\mathrm{gam} * \mathrm{a}$

else begin $\%$ neumann at $x_{-} 1$

$a b c:=-\operatorname{sub}(x i=0, h * d f(v(1), x))+\operatorname{gam} * a ;$

$l b c:=2 * a b c-r b c$;

end;

$\operatorname{gd}:=(r b c-l b c) / h^{\wedge} 2+\operatorname{solg}($ deq,$x i)$;

$g(j):=g(j)+g d$;

$\mathrm{v}(j):=\mathrm{v}(j)+\mathrm{h}^{\wedge} 2 * \operatorname{solv}(-\mathrm{deq}+\mathrm{gd}, \mathrm{xi})+\mathrm{xi} *(\mathrm{rbc}+\mathrm{lbc}) / 2$;

ok: $=$ if $(\mathrm{deq}=0)$ and $(\mathrm{rbc}=0)$ and $(\mathrm{lbc}=0)$ then ok else 0 ; end; 
72 showtime;

73 end until ok=1;

74

75 end;

\section{References}

[1] J. Carr. Applications of centre manifold theory, volume 35 of Applied Math. Sci. Springer-Verlag, 1981. C669

[2] J. Carr and R. G. Muncaster. The application of centre manifold theory to amplitude expansions. II. Infinite dimensional problems. J. Diff. Eqns, 50:280-288, 1983. C669

[3] T. MacKenzie and A. J. Roberts. The dynamics of reaction diffusion equations lead to a holistic discretisation. In R. L. May, G. F. Fitz-Gerald, and I. H. Grundy, editors, EMAC 2000 Proceedings. Proceedings of the fourth biennial Engineering Mathematics and Applications Conference, pages 199-202, 2000. C665

[4] T. Mackenzie and A. J. Roberts. Holistic finite differences accurately model the dynamics of the Kuramoto-Sivashinsky equation. ANZIAM J., 42(E):C918-C935, 2000. [Online] http://anziamj.austms.org.au/V42/CTAC99/Mack. C665

[5] National Physical Laboratory. Modern Computing Methods, volume 16 of Notes on Applied Science. Her Majesty's Stationary Office, 1961. C668

[6] A. J. Roberts. Low-dimensional modelling of dynamics via computer algebra. Computer Phys. Comm., 100:215-230, 1997. C682 
[7] A. J. Roberts. Holistic discretisation ensures fidelity to Burgers' equation. Applied Numerical Modelling, 37:371-396, 2001. C665, C667, C668, C671, C672, C673, C676

[8] A. J. Roberts. Holistic projection of initial conditions onto a finite difference approximation. Computer Physics Communications, 142:316-321, 2001. C665

[9] A. J. Roberts. A holistic finite difference approach models linear dynamics consistently. Mathematics of Computation, 72:247-262, 2003. [http://arXiv.org/abs/math.NA/0003135]. C665, C666, C667, C668, C673 